\title{
Hip Fracture in the Setting of Limited Life Expectancy: The Importance of Considering Goals of Care and Prognosis
}

\author{
C. Bree Johnston, MD, MPH, Amanda Holleran, MD, Thuan Ong, MD, MPH, \\ Ursula McVeigh, MD, and Elizabeth Ames, $\mathrm{MD}^{5}$
}

\begin{abstract}
Importance: Many older patients with a limited life expectancy experience fragility fracture of the hip, and this event is associated with increased risk of premature mortality, functional decline, and institutionalization. The treating team, in collaboration with patients and their families, must determine whether a surgical or conservative approach is in the patient's best interest when a patient has limited life expectancy.

Observation: Goals of care discussions appear to be rare in the setting of fragility fracture. The urgent nature of the problem makes such discussions challenging. We believe that many physicians have not considered goals of care discussions to be a standard component of fragility fracture management.

Conclusions: We propose that physicians caring for patients with limited life expectancy and fragility fracture of the hip should initiate a goals of care discussion to help determine whether operative repair will be the most patient-centered approach. Training on conducting goals of care discussions should be a standard part of surgical training programs. Goals of care discussions should include prognosis, patient values and preferences, pain, likelihood for functional recovery, and burdens and benefits of surgical versus nonsurgical management. Multidisciplinary input is required, and many patients will benefit from geriatric and/or palliative care team involvement.
\end{abstract}

Keywords: goals of care; hip fracture; limited life expectancy

\section{Background}

G ERIATRIC HIP FRACTURES are a mark of fragility in terms of both bone quality and patient characteristics. Twenty percent or more of all geriatric patients presenting with a lowenergy hip fracture will die within a year. ${ }^{1}$ Goals of care discussions appear to be rare in this setting, despite hip fracture as a potential "prompting event.", ${ }^{, 3}$ Some authors argue that nuanced end-of life discussions are an increasingly important competency for orthopedists to master, given the high population of frail elders who experience fragility fractures. ${ }^{2,4}$

Some patients with advanced illness, untenable operative risks, or comfort-oriented goals of care would benefit from conservative management. Unfortunately, the treating team has little evidence to guide when this approach should be recommended. ${ }^{5}$

Operative care has been the standard treatment for hip fracture for a very long time. The "fix-it" culture in surgery pre- sumes that a surgical intervention will fix the presenting problem. This can work well with acute illness or injury in a patient who is otherwise healthy. This model works much less well in a seriously ill patient who cannot be "fixed" and faces trade-offs in terms of risk and quality of life, regardless of whether operative or nonoperative management is chosen ${ }^{6,7} \mathrm{In}$ these patients, an outcomes-based approach that incorporates shared decision making and a discussion of goals of care should be considered the standard of care. Best communication practices in the setting of seriously ill older patients with emergency surgical conditions have been published., ${ }^{4,8}$

Culture is not the only barrier to making goals of care conversations a routine part of surgical practice. The fast pace of surgery and the improved outcomes with rapid management can make it challenging to carve out time for a lengthy discussion. These discussions are particularly challenging when patients or families present with anxiety, cognitive impairment, and family conflict. Very few surgeons are trained in how to

\footnotetext{
${ }^{1}$ Department of Medicine, University of Arizona College of Medicine, Tucson, Arizona.

${ }^{2}$ Orthopedics and Rehabilitation, University of Vermont College of Medicine, Burlington, Vermont.

${ }^{3}$ Geriatrics, University of Washington, Seattle, Washington.

${ }^{4}$ Providence Alaska Medical Group, WWAMI Regional Division, University of Washington School of Medicine, Anchorage, Alaska.

${ }^{5}$ Department of Orthopedics and Rehabilitation, University of Vermont College of Medicine, Burlington, Vermont.

Accepted April 25, 2018.
} 
navigate strong emotions and other challenges of communication. $^{4,8}$

\section{Methods}

We searched MEDLINE for peer-reviewed English language articles from 2000 to 2017 with major subject heading of hip fracture and prognosis. We also searched hip fracture and palliative care, hip fracture and hospice, and hip fracture and nonoperative management. Our primary approach is drawn from review of clinical practice guidelines, information about prognosis and functional recovery based on the literature, and our personal experiences in a range of treatment settings. Our focus is to provide general best practice recommendations to providers involved in the care of lowenergy hip fracture patients.

\section{Results}

\section{Scope of the problem}

The lifetime risk of hip fracture for men and women over age 50 years in the United States is $5 \%$ and $10 \%$, respectively. ${ }^{9}$ Hip fracture is strongly age related: patients of age 85 years and older are 10 times more likely to sustain a low-energy hip fracture than those aged $65-69$ years. ${ }^{10,11}$ Although the annual incidence of hip fracture has declined in the past decade, the population at risk continues to increase, and patients experiencing hip fracture appear to be older and sicker. ${ }^{12,13}$ Annual Medicare spending on hip fracture can be as high as 20 billion dollars a year, and this is expected to rise as the baby boomer generation ages. $^{14}$

\section{Mortality after hip fracture}

Mortality after hip fracture is significant. A total of $1.6 \%$ of patients will die during the initial hospitalization and $\sim 20 \%$ of women and $37 \%$ of men will die within one year. ${ }^{9,12}$ There has been no statistically significant reduction in one year mortality rates over the past three decades despite improvement in surgical techniques. ${ }^{15}$

Risk factors for increased mortality rates after fragility fracture include age, illness burden, functional impairment, and cognitive impairment. ${ }^{9,16}$ Patients with advanced chronic disease have worse outcomes. For example, the six-month mortality rate for patients with end-stage dementia was $55 \%$ after hip fracture, compared with $12 \%$ for cognitively intact patients. ${ }^{17}$ In-hospital mortality rate for hospice patients with hip fracture has been reported to be $9 \% .^{18}$

The mortality rate associated with surgically repaired fragility fractures is well described. Much less is known about the mortality rates among nonoperative patients. ${ }^{9,16}$ One study showed no significant difference in 30-day mortality rates between patients treated operatively and nonoperatively with immediate mobilization. ${ }^{19}$ A Cochrane review of five randomized trials found no differences in medical complications, mortality rate, or long-term pain in conservative versus surgical therapy for hip fracture. ${ }^{5}$ Sixmonth mortality rates of patients who pursue nonoperative management range from $26 \%$ to $88 \%$ depending on the studied cohort. ${ }^{18-22}$ One study of hospice patients with fragility fracture indicates that $83 \%$ underwent surgical repair and had a median survival of 117 days, compared with a median survival of 26 days in the $17 \%$ who elected nonop- erative management. ${ }^{18}$ It is unclear how much the mortality rate difference reflects underlying disease versus what type of management was elected.

\section{Functional recovery and morbidity after hip fracture}

Most patients with fragility fracture of the hip will lose a level of function after fracture. ${ }^{5,13,23,24}$ One study estimated that one year after fracture, $42 \%$ of survivors fail to return to their prefracture mobility, $35 \%$ are incapable of walking independently, and $20 \%$ are unable to shop independently. ${ }^{13}$ Only $50 \%$ of patients will regain full ability to perform activities of daily living, and only half of those will be independent with these tasks. Hip fractures are associated with a fourfold increase in the likelihood of requiring long-term nursing facility care and a twofold increase in the probability of entering into low-income status. ${ }^{24}$ There are data that functional outcomes are improved in patients treated operatively versus conservatively, with greater likelihood of ambulation, healing without deformity, and shorter hospital stay among patients treated operatively. 5,22

\section{Discussion}

Our suggested approach to hip fracture management in the patient with limited life expectancy is outlined in Figure 1.

The first step is to consider life expectancy. Forecasting an individual's mortality rate is beyond the scope of this article. However, a number of tools are available to assist practitioners in determining prognosis for patients with single diseases or with multimorbidity. Eprognosis is a web-based application that helps estimate mortality rate by directing users to the most appropriate published prognostic model. ${ }^{25,26}$

The vast majority of patients with a life expectancy of months to years will elect surgical repair of hip fracture with goals for rehabilitation. Patients with high likelihood of surgical mortality rate or who have a prefracture poor quality of life might consider their fracture a turning point for future goals of care.

Many patients with life expectancy of days to weeks should be considered for nonoperative repair, as they are less likely to have the time to experience the benefits of surgical repair. Those who are on hospice should discuss with their providers their goals for pain control and rehabilitation and whether they want to consider hospitalization and interruption in hospice services. Pain management is particularly relevant in these discussions.

The decision of whether or not to operate is often more nuanced for patients with life expectancy in the range of weeks to months. Factors other than prognosis are critical to consider and assess in every patient, but are particularly important for this group. A patient who has a life expectancy of months but who has a high burden of suffering from other diseases might consider a hip fracture as a time to transition to hospice and forego surgical interventions. Conversely, a patient with a short life expectancy who wants to achieve a goal such as a wedding or a graduation might elect surgery.

Hung, Dunn, and others note that discussion around goals of care should be initiated early in the course of treatment with a multidisciplinary approach, and we agree. ${ }^{2,27}$ Multiple team members are often involved in decision making for these patients-anesthesia providers, orthopedic surgeons, 


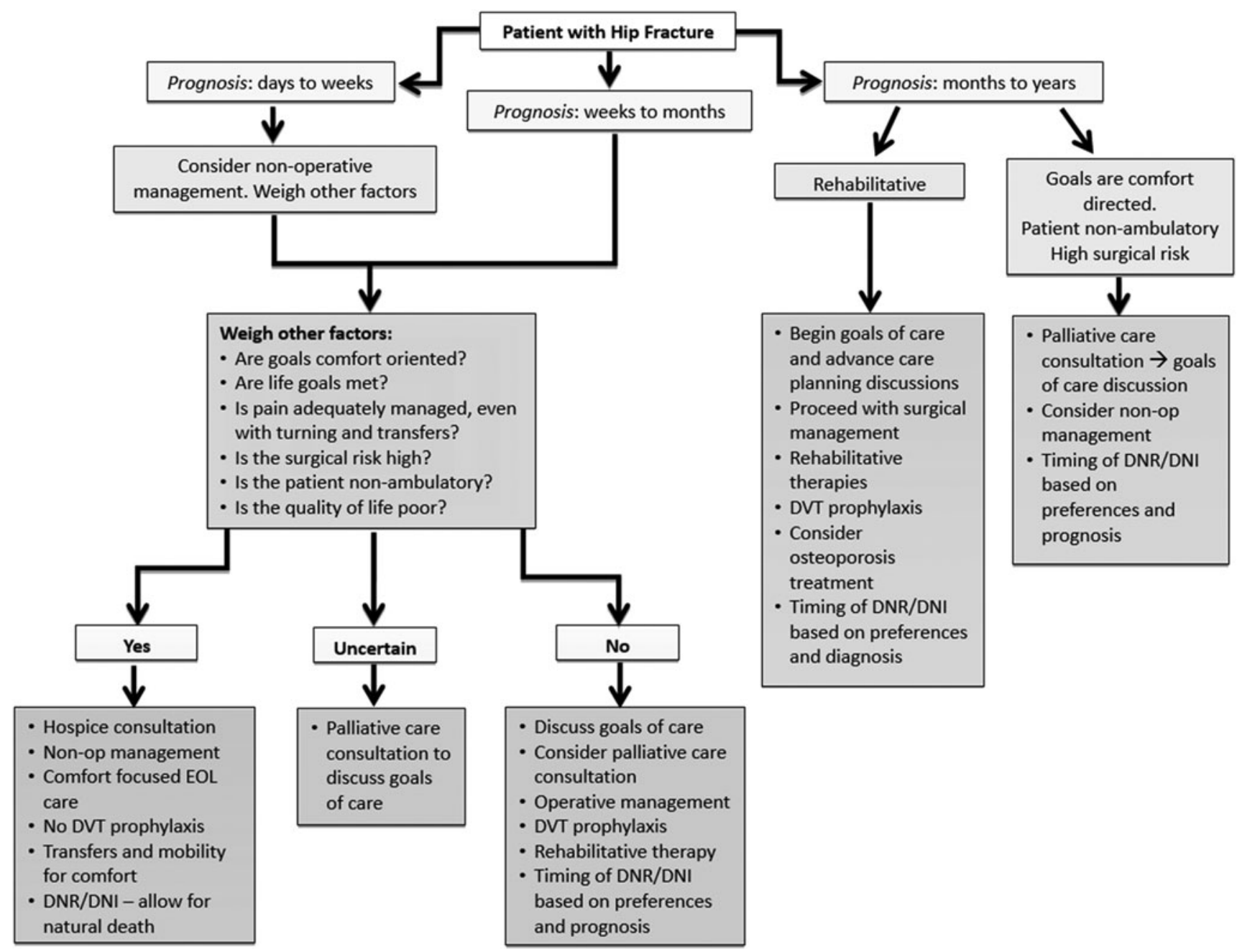

FIG. 1. Approach to patient with limited life expectancy and hip fracture.

hospitalists, geriatricians, rehabilitation disciplines, and others. Fairly straightforward goals of care discussions can usually be navigated by most practitioners with some basic training. Palliative care or geriatric services should be consulted for more complex or nuanced cases. Orthopedists, anesthesia providers, and other team members can benefit from formal training in conducting goals of care discussions, which is particularly important in institutions where palliative or geriatric providers are not available. Trust and flexibility between team members are a critical element in making these partnerships work optimally for patients and their families.

\section{Surgical versus nonsurgical options and management}

The surgical approach to various types of hip fracture are presented in Figure 2 and will not be reviewed in more detail here.

The goal of surgery may be comfort rather than rehabilitation. Operative management may be necessary for pain control and to facilitate optimal nursing care, even among bed-bound patients. ${ }^{28}$ Regardless of treatment choice, healing from a hip fracture varies considerably in terms of how much pain occurs, particularly in patients who are nonambulatory prefracture. There is not good data on whether operative or nonoperative treatment is associated with less pain. For nonambulatory patients, the degree of pain at rest and with movement should be a key consideration in whether to proceed operatively or conservatively. Aggressive pain control with standing, not prn, pain medications, including acetaminophen as well as opioid analgesics should be part of every care plan. ${ }^{27,28}$ Particular attention should be given to pretreatment before movement, transfers, and physical therapy sessions.

No studies have investigated optimal treatment for patients undergoing nonoperative treatment. ${ }^{29}$ Based on our experience, nonoperative protocols can be useful, including protected weight bearing for a period of time with advancement of activity as tolerated, similar to operative care. Strict bed rest is not required; limited weight bearing is recommended for approximately six weeks. Patients with inoperable hip fractures will need a wheelchair with a reclining back and an elevating leg rest for the affected side.

Optimal positioning after hip fracture can minimize movement of the affected hip while continuing routine personal care. Many patients will be most comfortable with their leg in the position it was injured (i.e., internally or externally 


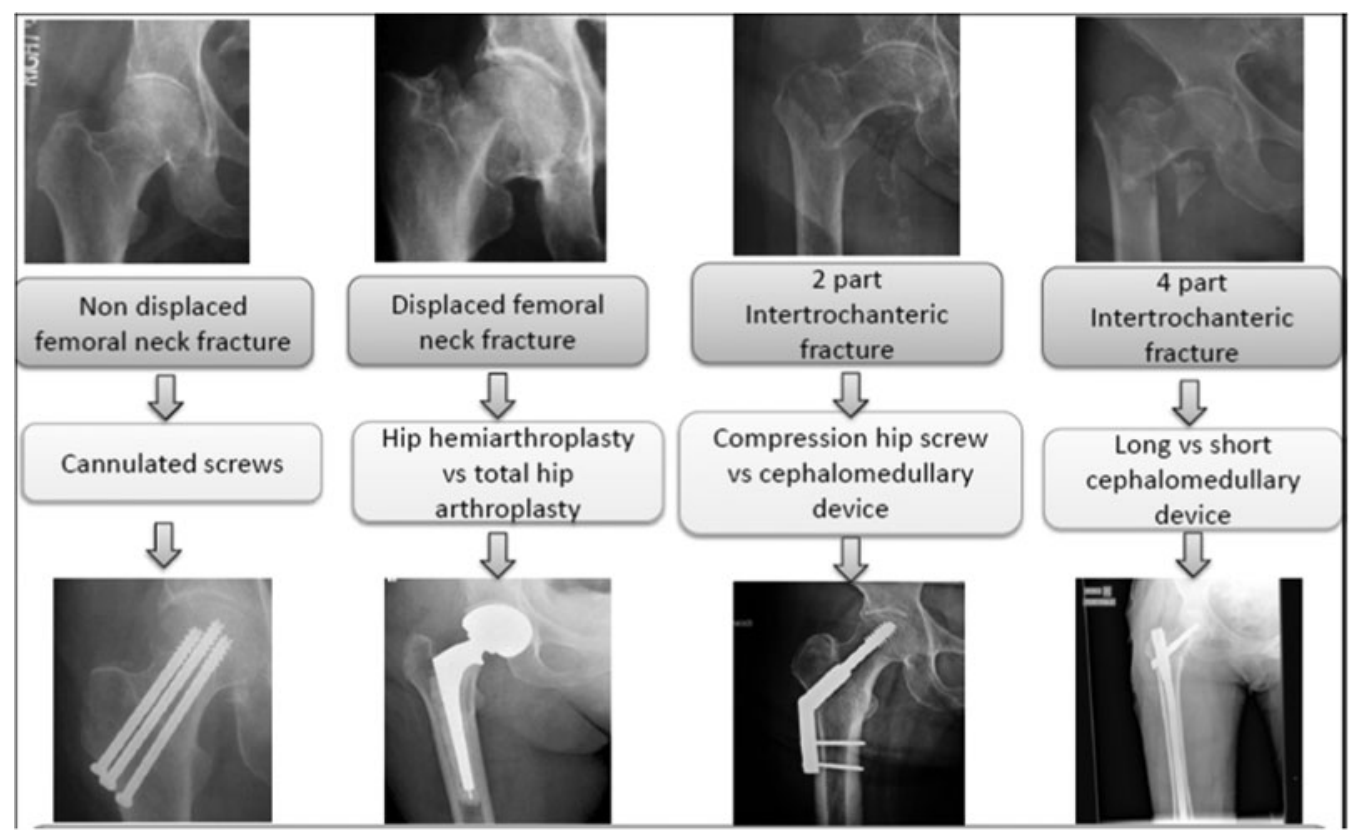

FIG. 2. Surgical approaches to hip fracture based on fracture type.

rotated). Using a pillow between the patient's legs before turning helps to keep the leg aligned and minimize internal rotation of the hip. In most situations, it is best not to attempt to bring the leg into a neutral position. Providing the majority of personal care while the patient is turned to the unaffected side will minimize the amount of time the patient will be on the side of his or her fracture. Education for nursing and other daily care providers is critical to achieve good results.

\section{Deep vein thrombosis prophylaxis}

The standard of care is to provide deep vein thrombosis (DVT) prophylaxis for a patient with hip fracture. However, in patients with very limited life expectancy and/or comfortoriented goals, foregoing DVT prophylaxis may be consistent with patient goals. This should be discussed explicitly for patients who have comfort rather than life-prolonging goals.

\section{Resuscitation status}

The issue of resuscitation status should be discussed with the patient and his or her surrogates. Some patients who opt for operative repair still have preferences for a "do-notresuscitate" (DNR) order, even in the operating room. Although hospital policies vary, many recognize DNR orders in the operating room. For the patient who chooses "full resuscitation" in the operating room only, it is important to ensure that policies are in place that ensures that the appropriate resuscitation order is written in the postoperative setting.

\section{Further fractures and osteoporosis}

Patients with limited life expectancy will not benefit from secondary medical osteoporosis treatment and should be excluded from clinical pathways that initiate osteoporosis treatment after fracture. The exact time to benefit for initiation of osteoporosis treatment is uncertain but likely to be about one year. ${ }^{30}$ All patients with hip fracture will benefit from fall prevention strategies and safety evaluation if any weight bearing is considered.

\section{Conclusion}

Fragility fractures are serious events with far ranging implications for both patients and families. As the population ages, it is increasingly vital that surgeons develop the skills to discuss surgery in the context of patient-centered outcomes, particularly when patients have limited life expectancy and a high burden of illness. Ensuring comfort, dignity, and achievement of patient-centered goals is critical regardless of the approach taken. Communicating with clarity around the burdens and benefits of both surgical and nonsurgical care plans is more likely to ensure patient-centered decisions at all levels of the patient care process. Patients and their families will elect the most appropriate approach to care when goals are discussed openly. A team-based approach including early input from geriatric or palliative care services may help facilitate high-quality discussions.

\section{Author Disclosure Statement}

No competing financial interests exist.

\section{References}

1. Panula J, Pihlajamaki H, Mattila VM, et al.: Mortality and cause of death in hip fracture patients aged 65 or older: A population-based study. BMC Musculoskelet Disord 2011; 12:105.

2. Dunn RH, Ahn J, Bernstein J: End-of-life care planning and fragility fractures of the hip: Are we missing a valuable opportunity? Clin Orthop Relat Res 2016;474:1736-1739.

3. Schonfeld TL, Stevens EA, Lampman MA, Lyons WL: Assessing challenges in end-of-life conversations with elderly patients with multiple morbidities. Am J Hosp Palliat Care 2012;29:260-267. 
4. Taylor LJ, Schwarze ML: Best practices: Targeting surgeon communication at the end of life. Ann Surg 2016;263:7-8.

5. Handoll HH, Parker MJ: Conservative versus operative treatment for hip fractures in adults. Cochrane Database Syst Rev 2008;16:CD000337.

6. Lynn J, DeGrazia D: An outcomes model of medical decision making. Theor Med 1991;12:325-343.

7. Kruser JM, Nabozny MJ, Steffens NM, et al.: "Best Case/ Worst Case": Qualitative evaluation of a novel communication tool for difficult in-the-moment surgical decisions. J Am Geriatr Soc 2015;63:1805-1811.

8. Cooper Z, Koritsanszky LA, Cauley CE, et al.: Recommendations for best communication practices to facilitate goal-concordant care for seriously ill older patients with emergency surgical conditions. Ann Surg 2016;263:1-6.

9. Frost SA, Nguyen ND, Center JR, et al.: Excess mortality attributable to hip-fracture: A relative survival analysis. Bone 2013;56:23-29.

10. Ensrud KE: Epidemiology of fracture risk with advancing age. J Gerontol A Biol Sci Med Sci 2013;68:1236-1242.

11. Samelson EJ, Zhang Y, Kiel DP, et al.: Effect of birth cohort on risk of hip fracture: Age-specific incidence rates in the Framingham Study. Am J Public Health 2002;92: 858-862.

12. Brauer CA, Coca-Perraillon M, Cutler DM, Rosen AB: Incidence and mortality of hip fractures in the United States. JAMA 2009;302:1573-1579.

13. Auais M, Morin S, Nadeau L, et al.: Changes in frailtyrelated characteristics of the hip fracture population and their implications for healthcare services: Evidence from Quebec, Canada. Osteoporos Int 2013;24:2713-2724.

14. Burge R, Dawson-Hughes B, Solomon DH, et al.: Incidence and economic burden of osteoporosis-related fractures in the United States, 2005-2025. J Bone Miner Res 2007;22: 465-475.

15. Mundi S, Pindiprolu B, Simunovic N, Bhandari M: Similar mortality rates in hip fracture patients over the past 31 years. Acta Orthop 2014;85:54-59.

16. Berry SD, Samelson EJ, Bordes M, et al.: Survival of aged nursing home residents with hip fracture. J Gerontol A Biol Sci Med Sci 2009;64:771-777.

17. Morrison RS, Siu AL: Survival in end-stage dementia following acute illness. JAMA 2000;284:47-52.

18. Leland NE, Teno JM, Gozalo P, et al.: Decision making and outcomes of a hospice patient hospitalized with a hip fracture. J Pain Symptom Manage 2012;44:458-465.

19. Jain R, Basinski A, Kreder HJ: Nonoperative treatment of hip fractures. Int Orthop 2003;27:11-17.
20. Yoon BH, Baek JH, Kim MK, et al.: Poor prognosis in elderly patients who refused surgery because of economic burden and medical problem after hip fracture. J Korean Med Sci 2013;28:1378-1381.

21. Gregory JJ, Kostakopoulou K, Cool WP, Ford DJ: One-year outcome for elderly patients with displaced intracapsular fractures of the femoral neck managed non-operatively. Injury 2010;41:1273-1276.

22. Ooi LH, Wong TH, Toh CL, Wong HP: Hip fractures in nonagenarians-A study on operative and non-operative management. Injury 2005;36:142-147.

23. Beaupre LA, Jones CA, Johnston DW, et al.: Recovery of function following a hip fracture in geriatric ambulatory persons living in nursing homes: Prospective cohort study. J Am Geriatr Soc 2012;60:1268-1273.

24. Tajeu GS, Delzell E, Smith W, et al.: Death, debility, and destitution following hip fracture. J Gerontol A Biol Sci Med Sci 2014;69:346-353.

25. Yourman LC, Lee SJ, Schonberg MA, et al.: Prognostic indices for older adults: A systematic review. JAMA 2012; 307:182-192.

26. Lee S, Smith A, Widera E, et al.: https://eprognosis.ucsf. edu/index.php (Last accessed April 5, 2018).

27. Hung WW, Egol KA, Zuckerman JD, Siu AL: Hip fracture management: Tailoring care for the older patient. JAMA 2012;307:2185-2194.

28. Morrison RS, Magaziner J, McLaughlin MA, et al.: The impact of post-operative pain on outcomes following hip fracture. Pain 2003;103:303-311.

29. Neuman MD, Fleisher LA, Even-Shoshan O, et al.: Nonoperative care for hip fracture in the elderly: The influence of race, income, and comorbidities. Med Care 2010;48: 314-320.

30. Lyles KW, Colon-Emeric CS, Magaziner JS, et al.: Zoledronic acid and clinical fractures and mortality after hip fracture. N Engl J Med 2007;357:1799-1809.

Address correspondence to:

C. Bree Johnston, MD, MPH

Division of Geriatrics, General Internal Medicine, and Palliative Medicine

Department of Medicine

University of Arizona College of Medicine

150 North Campbell Avenue

PO Box 245027

Tucson, AZ 85724-5027

E-mail: bree.johnston@bannerhealth.com 